\title{
Mejoramiento de las Propiedades de Tensión en WPC de LDPE: HIPS/Fibra Natural Mediante Entrecruzamiento con DCP
}

\author{
Arnoldo Emilio Delgado Tobón \\ Departamento de Ingeniería, Universidad Militar Nueva Granada, UMNG \\ Facultad de Ingeniería, Escuela Colombiana de Ingeniería Julio Garavito, ECIJG \\ William Arnulfo Aperador Chaparro \\ Facultad de Ingeniería, Universidad Militar Nueva Granada, UMNG \\ William Gómez Rivera \\ Facultad de Ingeniería, Universidad Militar Nueva Granada, UMNG
}

\begin{abstract}
Resumen: Fibra de Retamo Liso (telinemonspessulana) fue mezclada en una matriz polimérica formada por: polietileno de baja densidad (LDPE) y poliestireno de alto impacto (HIPS) en relación 70:30 respectivamente. La fibra natural fue sometida a reducción de tamaño entre 1700 y $850 \mu \mathrm{m}$; se le realizó tratamiento superficial de mercerización; el contenido de humedad fue $0,17 \%$. El análisis termo gravimétrico (TGA) muestra que la fibra tratada tiene estabilidad de $200,53{ }^{\circ} \mathrm{C}$. La elaboración del compuesto se realizó en mezclador interno y la técnica de obtención de las probetas fue mediante moldeo por compresión. Se evaluó el efecto del agente entrecruzante peróxido de dicumilo (DCP) en compuestos con varios porcentajes de fibra: $0 ; 10 ; 25 ; 50 \mathrm{phr}$. La adición de DCP (1 phr) en los compuestos, mejora significativamente las propiedades de tensión con respecto a similares concentraciones de fibra; sin embargo, esta diferencia en los valores de las propiedades disminuye progresivamente cuando la cantidad de fibra se aproxima a $50 \mathrm{phr}$. El aumento en la concentración de fibra afecta negativamente las propiedades de tensión, con excepción del Módulo de Elasticidad y la Resistencia a la Fluencia. La causa principal es originada por la baja adhesión interfacial fibra natural-matriz polimérica, claramente evidenciada en las imágenes SEM.
\end{abstract}

Palabras-clave: TelineMonspessulana, compuesto de plástico y madera (WPC), polietileno de baja densidad, poliestireno de alto impacto, peróxido de dicumilo.

\section{Improvement of Properties of Tension in WPC of LDPE: HIPS/ Natural Fiber Through Crosslinking with DCP}

Abstract: Straight Retamo Fiber (telinemonspessulana) was mixed in a polymer matrix formed by low density polyethylene (LDPE) and high impact polystyrene (HIPS) in a 70:30 ratio. The natural fiber was subjected to size reduction between 1700 and $850 \mu \mathrm{m}$; surface treatment mercerization was performed; the moisture content was $0.17 \%$. The thermo gravimetric analysis (TGA) showed that the treated fiber has stability at $200.53{ }^{\circ} \mathrm{C}$. The preparation of the compound was performed in an internal mixer with the samples being obtained by compression molding. The effect of crosslinking agent was evaluated: dicumyl peroxide (DCP) in compounds with various percentages of fiber: 0 ; 10; 25; $50 \mathrm{phr}$. The addition of DCP (1 phr) in the compounds significantly improved tensile properties compared to similar concentrations of fiber; however, this difference in property values progressively decreased when the amount of fiber approximated $50 \mathrm{phr}$. The increase in fiber concentration negatively affected the tensile properties, except for the modulus of elasticity and fluence resistance. The main cause is the low interfacial adhesion between the natural fiber and the polymer matrix, clearly evidenced in the SEM images.

Keywords: TelineMonspessulana, wood plastic composite (WPC), low density polyethylene, high impact polystyrene, dicumyl peroxide.

\section{Introducción}

En las últimas decadas las fibras naturales han sido consideradas como alternativa de refuerzo en compuestos poliméricos. Estas presentan buenas propiedades mecánicas, son biodegradables, reciclables, de bajo costo y baja densidad ${ }^{[1,2]}$. Sus principales desventajas en la elaboración y desempeño de compuestos poliméricos son: absorción de agua, formación de agregados, inestabilidad térmica, incompatibilidad fibra (hidrofílica)-polímero (hidrofóbica). Las características, funcionalidades y eficiencias de procesamiento son determinadas por la composición química y morfológica, las cuales varían según especie y condiciones de 
cultivación. Las fibras naturales están compuestas principalmente por: celulosa, hemicelulosa, pectina y lignina ${ }^{[3,4]}$. Generalmente, las fibras contienen $60-80 \%$ de celulosa y $5-20 \%$ de lignina ${ }^{[1,2]}$.

A pesar de que hay suficiente investigación publicada acerca del poder reforzante de la fibra natural en compuestos plásticos WPC (Wood Plastic Composite), no existe consenso acerca de las condiciones ideales que deben cumplir las fibras para que su efecto reforzante sea óptimo, debido a la cantidad de variables o características inherentes como especie vegetal, composición química, tamaño de partícula, morfología, tratamiento superficial y concentración en la mezcla. Por ejemplo, Lee et al. ${ }^{[5]}$ afirman que la resistencia a la tracción óptima es de 33,0 MPa en un compuesto de PP/Kenaf al 40\%; Rosário et al. ${ }^{[6]}$ mencionan que la misma propiedad es $34,4 \mathrm{MPa}$ para PP/ Sisal al 30\% indicando disminución del $9 \%$ con respecto a la tracción del PP virgen.

Spinace et al. ${ }^{[7]}$, analizaron WPCs con $20 \%$ de fibra de PP/Sisal y PP/Curauá en similares condiciones de procesamiento, hallaron que los compuestos con fibra de Curauá tienen mayor resistencia a la tracción, y en sentido opuesto, los compuestos con fibra de sisal tienen mayor resistencia al impacto. También se encuentran distintos resultados acerca de la relación entre el tamaño de partícula y el módulo de Young, el valor más alto fué de $2455 \mathrm{MPa}$ para partículas de 500-1000 $\mu \mathrm{m}$ en compuestos de HDPE/Bark al $60 \%$ según publica Ngu et al. ${ }^{[8]}$. Análisis similar reportaron Chen et al. ${ }^{[9]}$ en WPC de HDPE/madera, el valor fué $2218 \mathrm{MPa}$ con fibras de longitud mayor $1180 \mu \mathrm{m}$. Balam-Cocom et al. ${ }^{[10]}$. hallaron resultados opuestos al estudiar compuestos de PP/Piña de Henequen al 20\%, el valor mayor del módulo fué $650 \mathrm{MPa}$ con tamaño de partícula de $149 \mu \mathrm{m}$.

La relación L/D (longitud/diámetro) también afecta el módulo de elasticidad, por ejemplo en WPC de policaprolactona/almidón/sisal, al aumentar la razón L/D desde 76 hasta 126, el valor de la propiedad se incrementa desde 116 hasta $272 \mathrm{MPa}^{[11]}$.

La mercerización promueve la adhesión interfacial fibra-polímero, es un tratamiento químico superficial con hidróxido de sodio que aumenta el área superficial de la fibra mediante la remoción de materiales que contienen lignina $^{[12,13]}$. La concentración de soda cáustica, la temperatura y el tiempo de remojo de la fibra natural que mejoran las propiedades mecánicas de WPC son variables, prácticamente cada grupo de investigadores llegan a resultados diferentes, inclusive para un tipo de fibra similar. Por ejemplo, Liu et al. ${ }^{[14]}$ trataron fibra de jute con $\mathrm{NaOH}$ al $2 \%$, durante tres horas a temperatura ambiente, para fabricar compuestos con succinato de polibutileno, Khan et al. afirman que las condiciones óptimas para el mismo tipo de fibra en compuestos con PP son: concentración de $\mathrm{NaOH}$ al 20\%, temperatura $0{ }^{\circ} \mathrm{C}$ y tiempo de remojo 1 hora $^{[15]}$. Pires et al. lograron mejorar las propiedades mecánicas de una matriz epóxica con fibras de jute tratadas con $\mathrm{NaOH}$ al $5 \%$ a temperatura ambiente durante 6 horas ${ }^{[16]}$.

En la presente investigación se elaboró un WPC con matriz polimérica de LDPE/HIPS y fibra natural de Retamo Liso. Generalmente la mayoría de las mezclas entre polímeros son inmiscibles, la combinación LDPE/ HIPS no es la excepción, presentando baja adhesión interfacial ${ }^{[17]}$. Cuando los polímeros son inmiscibles se necesita la compatibilización para obtener una sinergia de las propiedades mecánicas de cada polímero. La mezcla LDPE/HIPS puede compatibilizarse con la adición de un copolímero de bloque HPB-b-PS, que mejora la adhesión entre las fases ${ }^{[17]}$.

La compatibilización entre poliolefinas y polímeros con insaturación (doble enlace en la cadena polimérica) también se puede realizar mediante la incorporación de agentes de entrecruzamiento (compatibilización reactiva $)^{[17-20]}$.

La estrategia de compatibilización en esta investigación consiste en la incorporación de un peróxido orgánico como agente iniciador de radicales libres. El peróxido de dicumilo (DCP) reticula las cadenas de LDPE y HIPS, permitiendo la unión química entre las dos fases poliméricas. El entrecruzamiento cambia la naturaleza de la mezcla polimérica de termoplástica a termoestable. El DCP se descompone a $130{ }^{\circ} \mathrm{C}$ generando radicales libres que sustraen hidrógenos de la cadena de LDPE, en este sitio se forma un radical activo que forma un enlace con otro radical activo de la misma o diferente cadena ${ }^{[21,22]}$. El HIPS es un copolímero formado por bloques de polibutadieno (PB) y poliestireno (PS). La reacción de entrecruzamiento se produce mediante la acción de los radicales libres generados por el DCP los cuales rompen el doble enlace presente en las unidades polibutadieno (PB), generando en este sitio un radical activo que se unirá a otro radical activo de la misma o diferente cadena. Mokoena et al. analizaron el efecto del DCP sobre la resistencia a la tracción y el módulo de Young de WPC de LDPE/sisal, los compuestos formulados con $1 \%$ de peróxido incrementan en $70 \%$ las propiedades de tensión, para igual concentración de fibra ${ }^{[23]}$.

El objetivo de esta investigación es evaluar la influencia del contenido de fibra y el efecto del agente entrecruzante (DCP) sobre las propiedades mecánicas de WPC de LDPE/HIPS/ Retamo Liso.

\section{Parte experimental}

\section{Materiales}

La fibra natural de Retamo Liso (Teline monspessulana) fue sometida a tratamiento superficial con $\mathrm{NaOH}$ al $30 \% \mathrm{~m} / \mathrm{m}$, posteriormente fue mezclada con una matriz polimérica compuesta de polietileno de baja densidad (LDPE, MFI: 1,7-2,3 g/10 min fabricado por Ecopetrol ${ }^{\circledR}$ ) y poliestireno de alto impacto (HIPS, Emulprene 260, contenido de estireno 62,5$64,5 \%$, fabricado por Industrias Negromex INSA $^{\circledR}$ ). Los compuestos fueron reticulados con peróxido de dicumilo (Luperox ${ }^{\circledR}$ DCP, pureza: 98\%, suministrado por Arkema $^{\circledR}$ ). El lubricante externo formulado para evitar que los polímeros se adhieran a las paredes del mezclador interno fué acido esteárico (doble prensado, título 60, suministrado por industrias $\mathrm{DERSA}^{\circledR}$ ), finalmente en la elaboración de las mezclas se empleó como agente homogeneizador una mezcla de resinas de hidrocarburo alifáticas (Struktol 60NSF ${ }^{\circledR}$, de Struktol Company ${ }^{\circledR}$ ). Los 
reactivos empleados para la determinación del punto de gel: xileno, antioxidante Songonox ${ }^{\circledR} 1076$.

\section{Métodos}

\section{Caracterización y tratamiento de la fibra}

Análisis bromatológico: Se realizó en el laboratorio de Nutrición Animal de la Universidad Nacional de Colombia. Los resultados se reportan en la Tabla 1.

Molienda: La longitud de la fibra fue reducida hasta un tamaño de 0,85 a $1,7 \mathrm{~mm}$ en un molino de martillos NOGUEIRA $^{\circledR}$, modelo DPM Junior, velocidad 3500 y 2,2 $\mathrm{kW}$ de potencia.

Mercerización: La fibra fue sumergida en solución de $\mathrm{NaOH}$ al $30 \%$, durante 2 horas, a $23{ }^{\circ} \mathrm{C}$, con agitación manual cada 15 minutos.

Secado: Las fibras fueron colocadas en un horno con recirculación forzada Quincy Lab ${ }^{\circledR}$ 21-250 durante 23 horas a $100{ }^{\circ} \mathrm{C}$. El contenido de humedad final fue $0,17 \%$.

\section{Elaboración de compuestos}

Se realizaron ocho formulaciones, los valores fueron establecidos en partes por cien de resina (phr), donde el término resina se aplica a la suma de los polímeros (Tabla 2); las cantidades de lubricante externo, agente homogenizante y polímeros permanecieron constantes. Las variables fueron DCP (0 ó 1 phr) y nivel de fibra (10; 25; 50 phr).

Mezclado: Se empleó un mezclador interno tipo banbury formado por 2 rodillos triangulares, con torque máximo de $10 \mathrm{~N} / \mathrm{m}$ y capacidad de $60 \mathrm{~cm}^{3}$. Se agregaron los polímeros, el agente lubricante y posteriormente la fibra con el agente homogeneizador, cuya función es aglomerar de manera rápida la fibra con el polímero. El tiempo de mezclado fue 10 minutos. En las formulaciones con DCP, éste se adicionó 2 minutos antes de finalizar el ciclo. La temperatura de la mezcla fue: $125^{\circ} \mathrm{C}$.

Tabla 1. Análisis bromatológico fibra de Retamo Liso.

\begin{tabular}{lcc}
\hline \multicolumn{1}{c}{ Análisis } & \multicolumn{2}{c}{ Base (\%) } \\
\cline { 2 - 3 } & Húmeda & Seca \\
\hline Materia seca & & 59,9 \\
Fibra detergente neutro & 52,5 & 89,0 \\
Fibra detergente ácido & 36,5 & 61,9 \\
Lignina & 6,73 & 11,4 \\
Celulosa & 29,7 & 50,3 \\
Hemicelulosa & 16,0 & 27,1 \\
\hline
\end{tabular}

Prensado: El compuesto fue colocado en un molde de área: $15 \mathrm{~cm} \times 15 \mathrm{~cm}$ y espesor: $0,3 \mathrm{~cm}$. Las condiciones de operación de la prensa hidráulica: temperatura de los platos: $160 \pm 2{ }^{\circ} \mathrm{C}$, presión específica: $3,82 \mathrm{MPa}$, tiempo de prensado: 10 minutos. Durante este proceso se realiza el entrecruzamiento polimérico de los compuestos que contienen DCP.

\section{Métodos de análisis}

Microscopia Electrónica de Barrido (SEM): Las imágenes fueron tomadas con microscopio SEM, modelo NeosCope JCM 5000, la metalización de las muestras se realizó en un sputter Balzers ${ }^{\circledR}$ SDC 050 en condiciones de prevacio, con argón como gas de ataque (plasma) sobre una placa de oro-paladio. El voltaje de aceleración fue $30 \mathrm{kV}$.

Análisis Termogravimétrico (TGA): La fibra tratada fue caracterizada mediante análisis termogravimétrico en el equipo TGA-2050 T.A. Instruments ${ }^{\circledR}$; velocidad de calentamiento: $10{ }^{\circ} \mathrm{C} / \mathrm{min}$ desde $25{ }^{\circ} \mathrm{C}$ hasta $1000{ }^{\circ} \mathrm{C}$, atmósfera de $\mathrm{N}_{2}$.

Determinación del Punto de Gel: El grado (eficiencia) de entrecruzamiento se determinó aplicando el método A de la Norma ASTM D 2765-01 ${ }^{[24]}$. La prueba se aplicó a las probetas formuladas con DCP sometidas al proceso de

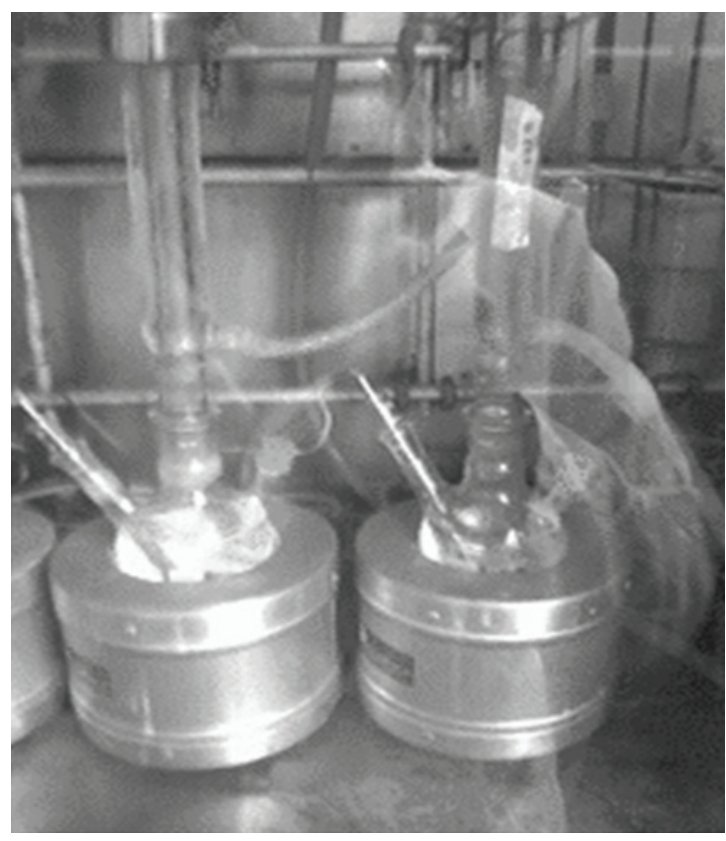

Figura 1. Montaje para determinar el punto de Gel.

Tabla 2. Formulaciones de WPC con base en 100 partes de resina.

\begin{tabular}{|c|c|c|c|c|c|c|c|c|c|}
\hline \multirow[t]{2}{*}{ Ingrediente } & \multirow[b]{2}{*}{ No Formula } & \multicolumn{4}{|c|}{ Sin DCP } & \multicolumn{4}{|c|}{ Con DCP } \\
\hline & & (1) & (2) & (3) & (4) & (5) & (6) & (7) & (8) \\
\hline LDPE & & 70,0 & 70,0 & 70,0 & 70,0 & 70,0 & 70,0 & 70,0 & 70,0 \\
\hline HIPS & & 30,0 & 30,0 & 30,0 & 30,0 & 30,0 & 30,0 & 30,0 & 30,0 \\
\hline A. Esteárico & & 1,0 & 1,0 & 1,0 & 1,0 & 1,0 & 1,0 & 1,0 & 1,0 \\
\hline Struktol $^{\circledR} 60$ NSF & & 3,0 & 3,0 & 3,0 & 3,0 & 3,0 & 3,0 & 3,0 & 3,0 \\
\hline Fibra Natural & & - & 10,0 & 25,0 & 50,0 & - & 10,0 & 25,0 & 50,0 \\
\hline DCP & & - & - & - & - & 1,0 & 1,0 & 1,0 & 1,0 \\
\hline
\end{tabular}


reticulado. El equipo consta de un balón con capacidad de $500 \mathrm{~cm}^{3}$, un condensador con entrada y salida de agua, manta de calentamiento y sensor de temperatura (Figura 1). El balón fué llenado con $300 \mathrm{~cm}^{3}$ de xileno y 2,5 gramos de antioxidante Songnox $1076^{\circledR} ; 0,300$ gramos de compuesto con tamaño de $0,150 \mathrm{~mm}$, fueron cubiertos en una malla de acero inoxidable 200 de la serie Tayler ${ }^{\circledR}$, posteriormente la muestra fue sumergida en xileno. Las condiciones del ensayo fueron: temperatura $143{ }^{\circ} \mathrm{C}$, tiempo de reflujo: 12 horas. La función del antioxidante es evitar que las cadenas poliméricas se entrecruzen debido a la oxidación.

Pruebas de Tensión: Se realizaron según la norma ASTM D 638-02 ${ }^{[25]}$, en el equipo universal Shimadzu ${ }^{\circledR}$ AGS-X, celda tipo $\mathrm{S}$ clase 1 de $10 \mathrm{kN}$; velocidad de desplazamiento $5 \mathrm{~mm} / \mathrm{min}$. Se usaron probetas tipo IV,

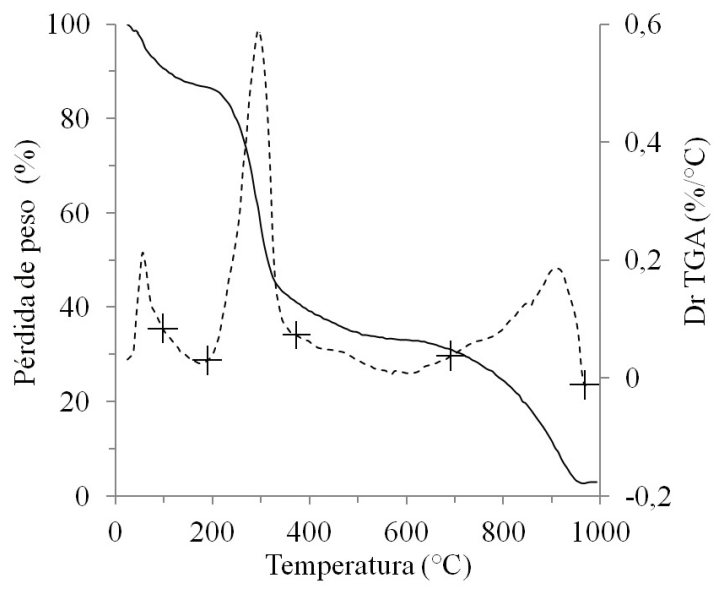

Figura 2. TGA de la fibra de Retamo Liso con tratamiento superficial.

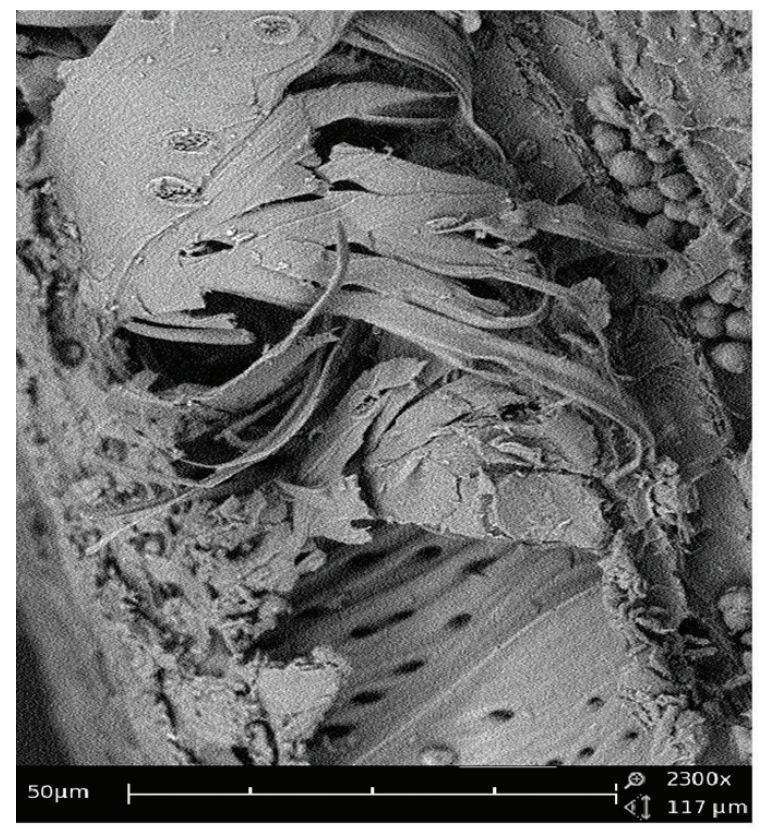

(a) cuatro por cada formulación. Condiciones del ensayo: temperatura $23{ }^{\circ} \mathrm{C}$ y humedad relativa $45 \%$.

\section{Resultados y Análisis}

\section{Fibra natural de Retamo Liso}

\section{Análisis bromatológico}

La literatura reporta que generalmente las fibras contienen $60-80 \%$ de celulosa, 5-20\% de lignina y hasta $20 \%$ de humedad ${ }^{[1]}$. Al comparar estos valores con los obtenidos en el análisis bromatológico para la fibra de Retamo Liso sin tratamiento superficial (Tabla 1), se observa que el contenido de celulosa es bajo, la cantidad de agua es alta, mientras que la lignina se encuentra dentro de los valores reportados.

\section{Estabilidad térmica de la fibra}

En el TGA (Figura 2) se observa pérdida inicial de masa en $101,76^{\circ} \mathrm{C}$, atribuible a la eliminación de agua. A $200,53{ }^{\circ} \mathrm{C}$ se inicia la degradación de la fibra vegetal, proceso que ocurre hasta $684,24^{\circ} \mathrm{C}$.

De acuerdo a la literatura, la lignina, algunos polisacáridos y ciertas sustancias inorgánicas pueden ser causantes de la continuidad en el proceso degradativo, que se presenta a temperaturas incluso por encima de $550{ }^{\circ} \mathrm{C}^{[26,27]}$.

Modificación superficial de la fibra de Retamo Liso (Morfología)

La mercerización remueve las impurezas en la superficie de la fibra de retamo liso y aumenta la porosidad de la partícula, según se aprecia en las imágenes SEM (Figura 3). Este proceso incrementa el área superficial y permite una mejor adhesión fibra/matriz, pues los espacios vacíos en la superficie promueven el enclavamiento mecánico. Liu et al. ${ }^{[14]}$ afirman que la

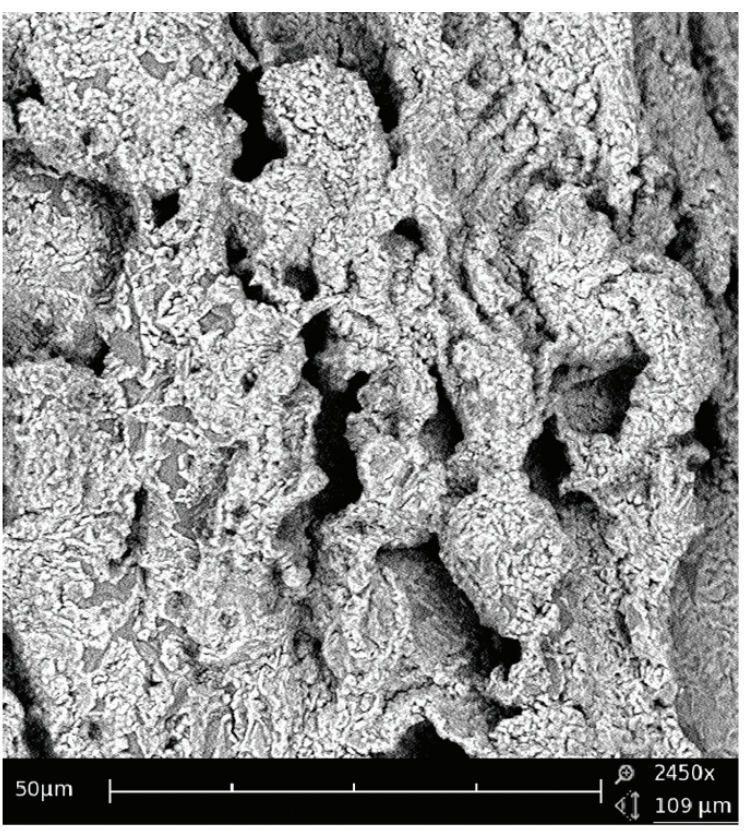

(b)

Figura 3. SEM de la superficie de la fibra Retamo Liso: a) sin tratamiento; b) con mercerización. 
mercerización cambia la estructura de la celulosa, lo cual probablemente origina fibras rígidas y fuertes de gran interés en la elaboración de WPC.

\section{Compuestos WPC}

\section{Determinación del contenido de gel}

El contenido de gel permite establecer la eficiencia en el proceso de curado o reticulado en aquellos compuestos formulados con DCP (Tabla 3). Las fracciones solubles son aquellas que no han sido entrecruzadas químicamente.

Los resultados permiten establecer que el entrecruzamiento fué parcial, generalmente los valores reportados por la literatura de poliolefinas reticuladas se encuentran entre $75-85 \%$, estudios adicionales serán necesarios para determinar si el contenido de fibra puede afectar la eficiencia de reticulación. El grado de entrecruzamiento puede elevarse mediante el incremento en la cantidad de DCP por ejemplo 1,5 phr o aumentando el tiempo de curado y/o la temperatura.

\section{Morfología de las zonas de fractura en los ensayos de tensión}

Fractografía de la mezcla polimérica LDPE/HIPS virgen: En la zona de fractura de la matriz polimérica sin DCP (Figura 4a) se observa no uniformidad en la superficie, hay formación de fibrillas y presencia de nódulos (segmento de polibutadieno del HIPS), se generan espacios vacíos. La presencia de fibrillas es debida al estiramiento longitudinal (deformación plástica) durante el ensayo de tensión. En conclusión es una fractura dúctil.

Tabla 3. Contenido de gel de las formulaciones con DCP.

\begin{tabular}{ccc}
\hline No. Fórmula & Fibra (phr) & Gel (\%) \\
\hline$(5)$ & 0,0 & 69,0 \\
$(6)$ & 10,0 & 67,0 \\
$(7)$ & 25,0 & 54,0 \\
$(8)$ & 50,0 & 59,0 \\
\hline
\end{tabular}

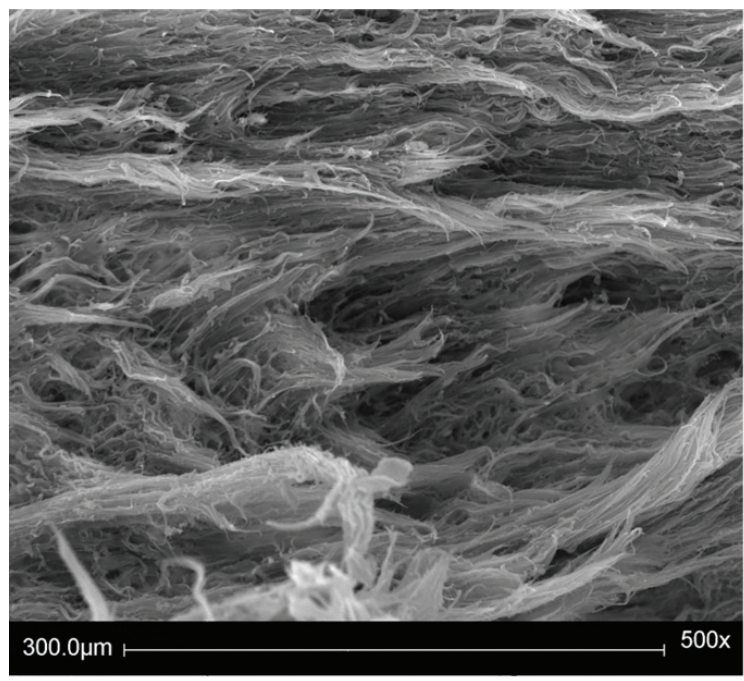

(a)
En la imagen (Figura 4b) se observa la superficie más homogénea, hay disminución de los nódulos, fibrillas y espacios vacíos. Claramente la adición de agente entrecruzante liga las cadenas, disminuyendo la deformación plástica.

Fractografía del material compuesto (WPC): Las imágenes SEM (Figura 5a-f) corresponden a la superficie fracturada de los compuestos con y sin DCP que contienen 10,25 y $50 \mathrm{phr}$ de fibra.

En todas las imágenes se evidencia la formación de espacios entre fibra y polímero. La baja adhesión interfacial fibra natural-matriz tiene gran incidencia en el desprendimiento de la fibra (pull out).

$\mathrm{Al}$ aumentar el contenido de fibra la concentración de espacios entre la fibra y el polímero también aumenta, generando puntos de falla los cuales disminuyen la resistencia a la tensión (Figura 6a, b). El DCP no tiene efecto sobre la disminución del pull out, es un peróxido cuya función específica es la reticulación de las cadenas poliméricas.

\section{Propiedades mecánicas}

Módulo de Young: $\mathrm{Al}$ incrementar la concentración de fibra, el módulo aumenta debido al carácter elástico propio de las fibras naturales. Las formulaciones que contienen DCP presentan mayores valores de la propiedad, pero la diferencia no es significativa cuando la concentración de fibra es cercana a $50 \mathrm{phr}$ (Figura 7a).

Resistencia a la tracción: La tracción disminuye al aumentar el contenido de fibra, no hay verificación del efecto reforzante de esta (Figura 7b). Una de las causas es la baja adhesión fibra-matriz observada en las imágenes SEM (Figura 5a-f), en ellas se aprecia la formación de micro grietas en la interfase fibra-polímero, las cuales fomentan la fractura del material. Los esfuerzos que actúan sobre la matriz se transmiten a las fibras a través de la interfase; cuando la adhesión interfacial es fuerte, las propiedades mecánicas del compuesto mejoran ${ }^{[28]}$. Igual que en las propiedades anteriores, los compuestos

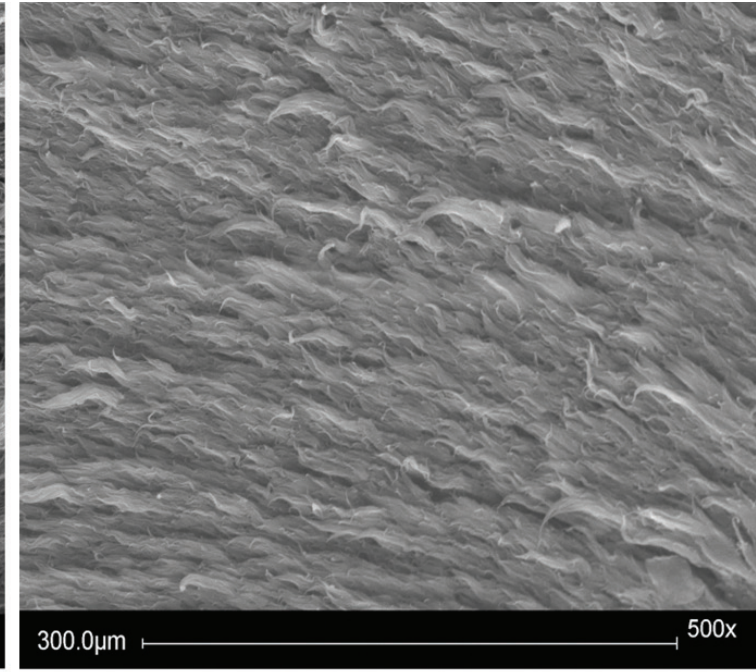

(b)

Figura 4. SEM de la superficie fracturada de la matriz polimérica virgen LDPE/HIPS: a) sin DCP formula (1); b) con DCP fórmula (5). 


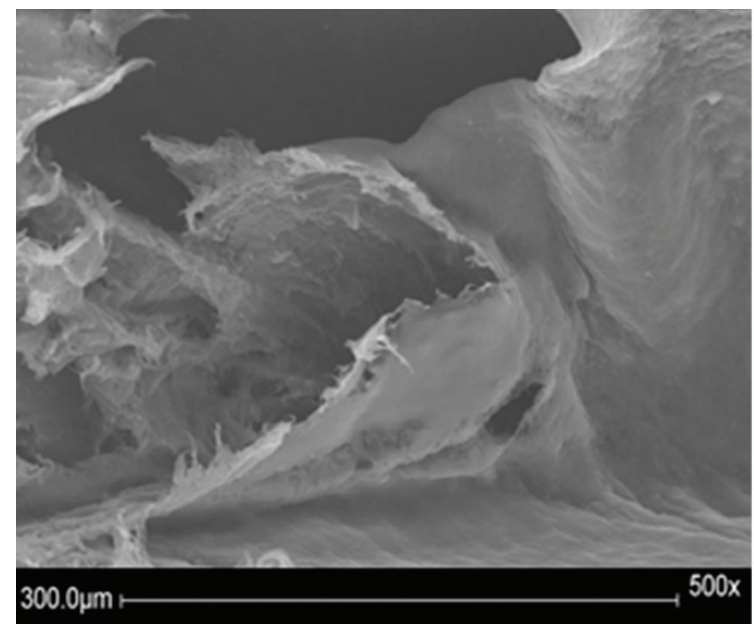

(a)

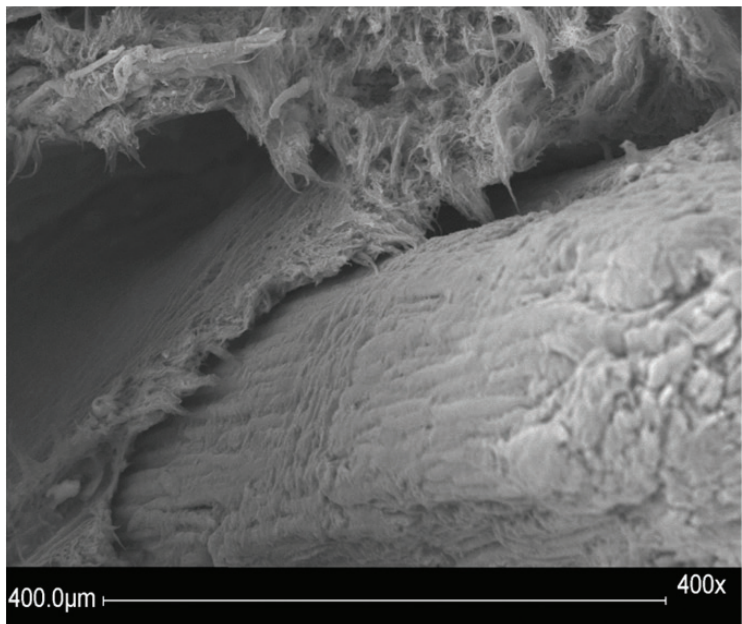

(c)

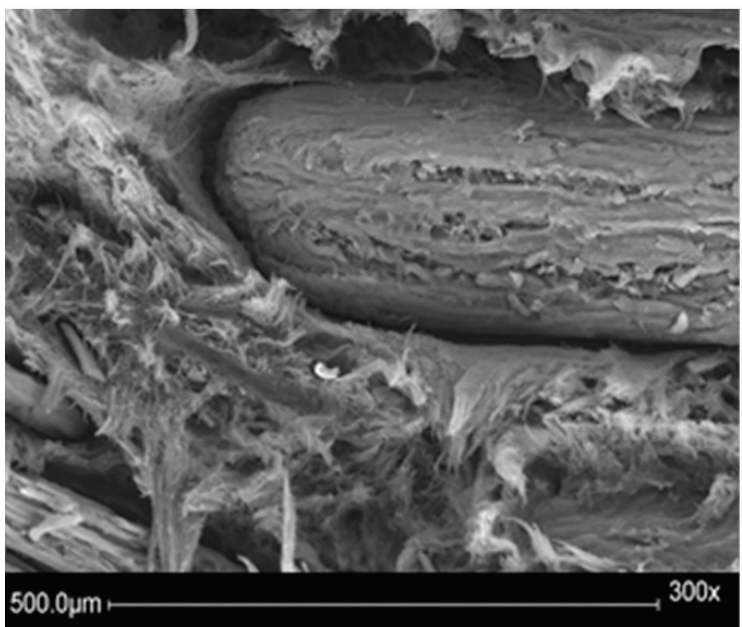

(e)

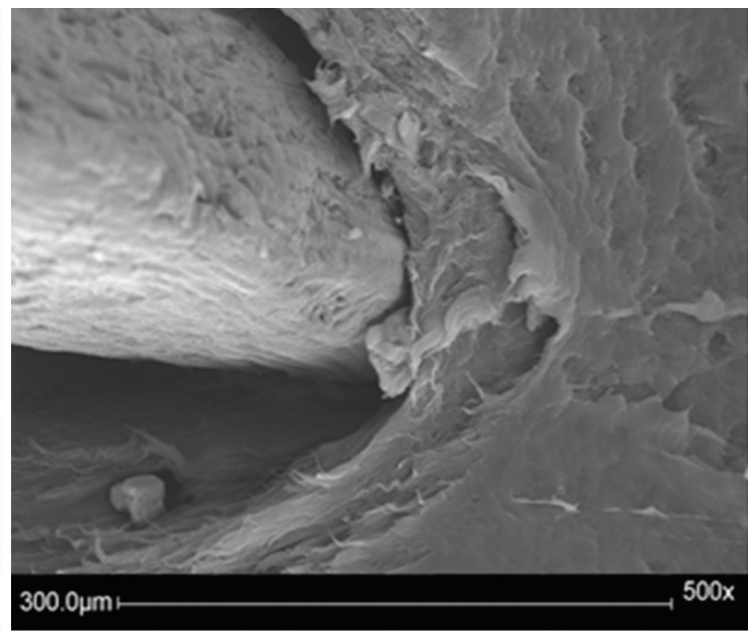

(b)

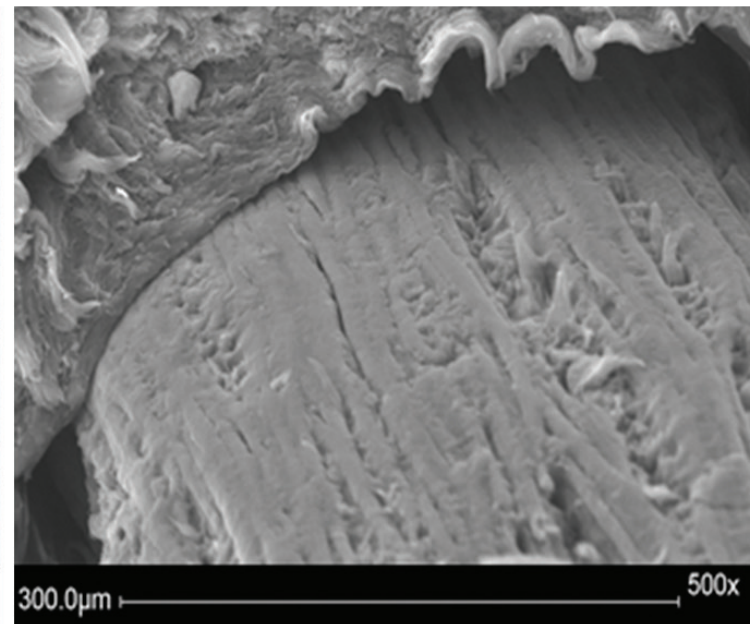

(d)

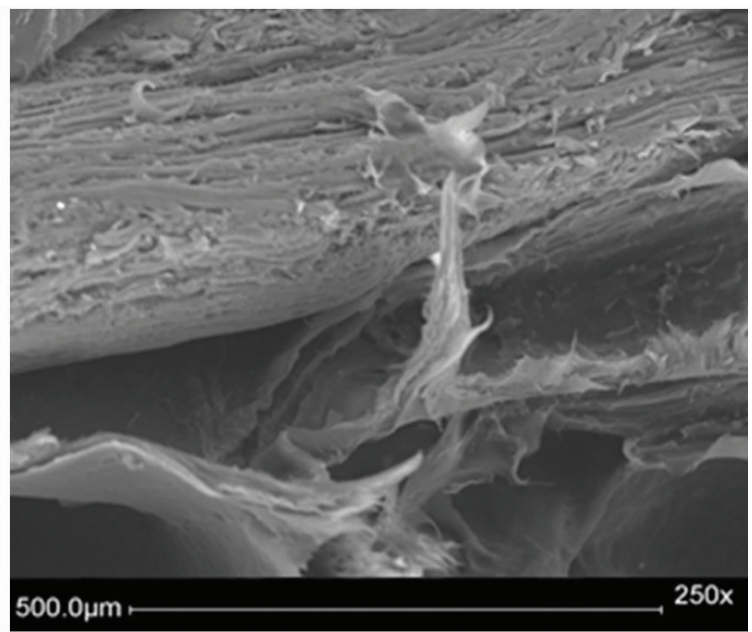

(f)

Figura 5. SEM de la superficie fracturada de WPC: a) 10 phr fibra, sin DCP; b) 10 phr de fibra, con DCP; c) 25 phr de fibra, sin DCP; d) $25 \mathrm{phr}$ de fibra, con DCP; e) $50 \mathrm{phr}$ de fibra sin DCP; f) $50 \mathrm{phr}$ de fibra con DCP. 


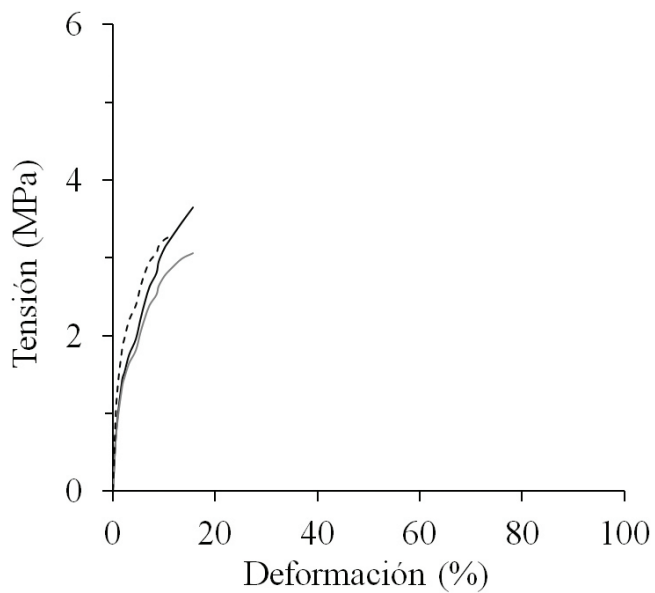

(a)

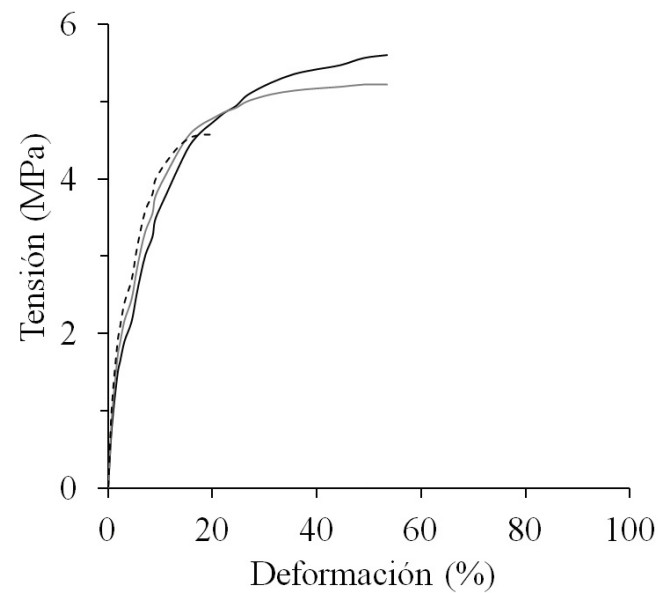

(b)

Figura 6. Ensayo de Tensión para los compuestos WPC: a) sin DCP; b) con DCP. Cantidad de fibra: 10 phr (—), 25 phr (-), $50 \mathrm{phr}(---)$.

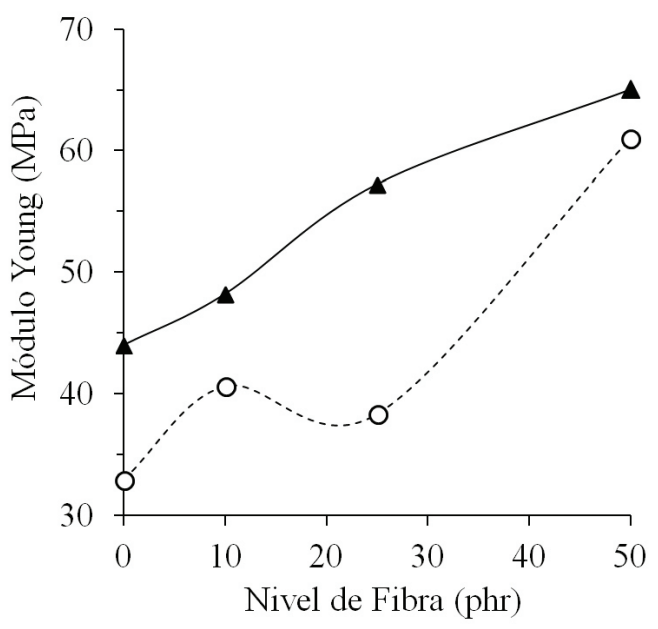

(a)

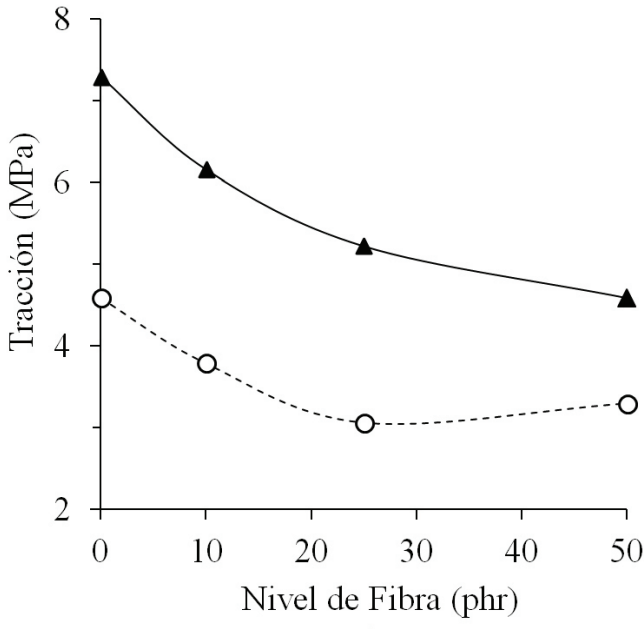

(b)

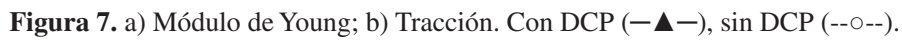

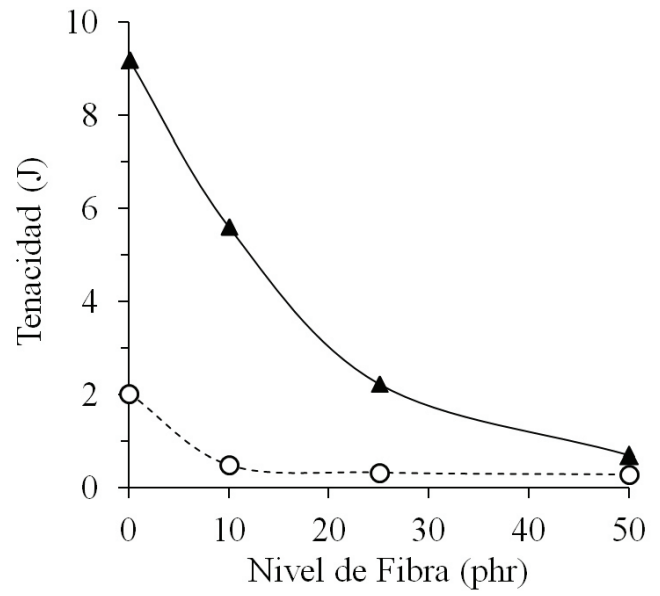

(a)

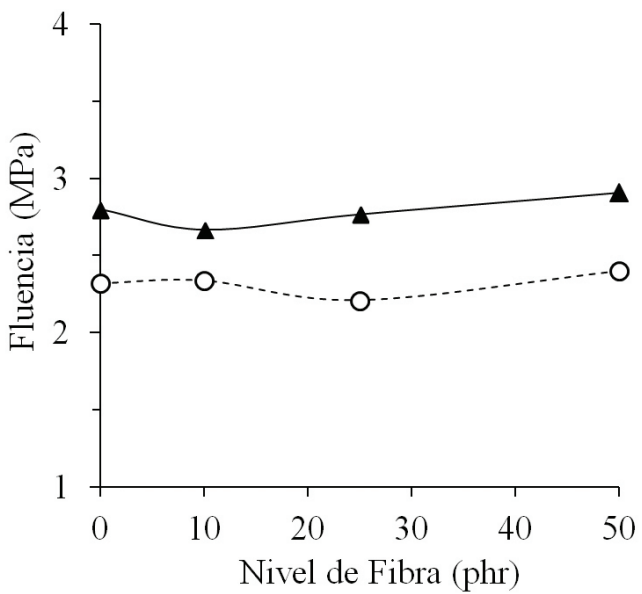

(b)

Figura 8. a) Tenacidad; b) Fluencia. Con DCP (-ム-), sin DCP (--०--). 
con DCP presentan valores más altos debido al entrecruzamiento de las cadenas poliméricas.

Tenacidad: El contenido de fibra disminuye la tenacidad del material, la baja adhesión interfacial fibrapolímero y los espacios vacíos entre estas fases inciden en el resultado (Figura 8a). Los espacios vacíos promueven la ruptura del material; se pueden apreciar en cada una de las imágenes SEM (Figura 5a-f). El valor de la tenacidad es mayor en los compuestos con DCP. Sin embargo, al incrementar el contenido de fibra el efecto del DCP no es significativo debido a la menor cantidad de polímero disponible para cubrir superficialmente la partícula de fibra.

Resistencia a la fluencia: Es mayor en los compuestos formulados con DCP e independiente de la concentración de fibra. El DCP aumenta la resistencia a la fluencia, pues al promover el entrecruzamiento confiere carácter elástico al material (Figura 8b).

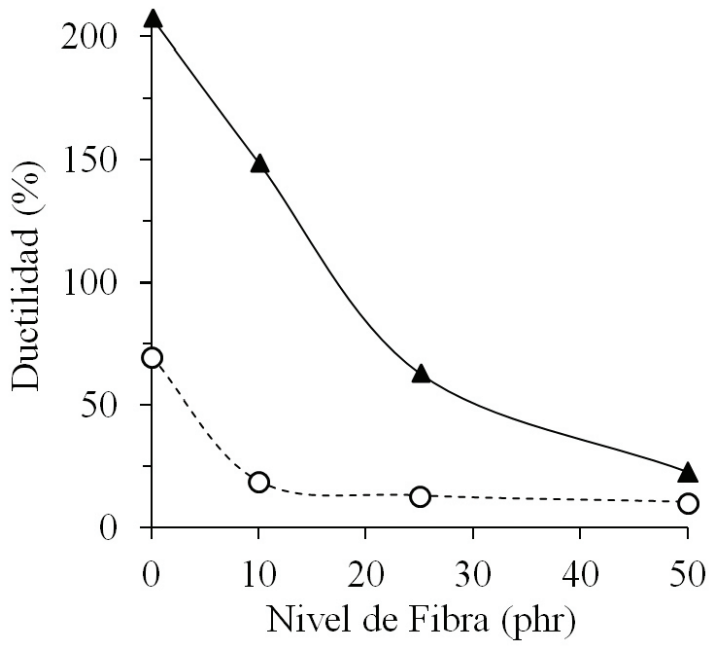

Figura 9. Ductilidad. Con DCP (- $\mathbf{\Delta - )}$, sin DCP (-- --).

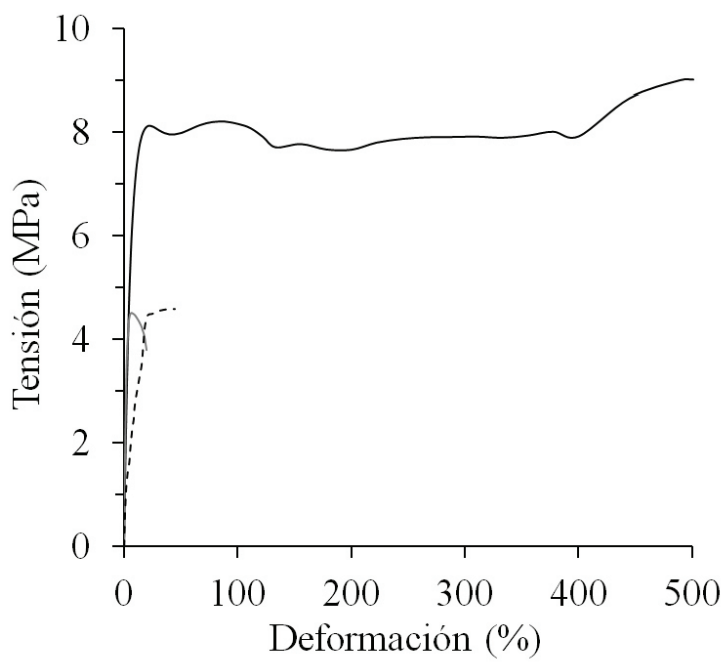

(a)
Ductilidad: Los compuestos con alto contenido de fibra presentan fractura frágil. La ductilidad decrece de manera rápida al aumentar el contenido de fibra. Realmente no se puede afirmar que hay aumento de la ductilidad en todas las mezclas entrecruzadas con DCP, con respecto a las no reticuladas (Figura 9).

La explicación de esta situación está soportada en los ensayos de tensión-deformación realizado a los polímeros HIPS, LDPE y LDPE/HIPS 70/30 (Figura 10a, b), se aprecia claramente que hay un efecto positivo al reticular el HIPS, la deformación del HIPS entrecruzado es $245 \%$ frente a un $20 \%$ sin reticular.

El HIPS está compuesto por dos fases inmiscibles las unidades polibutadieno (37\%) y poliestireno $(63 \%)$, por lo tanto al ligar las cadenas poliméricas se aumenta la resistencia del material. Mientras que para el LDPE el resultado hallado concuerda con la teoría, la deformación del reticulado es $445 \%$ frente a $500 \%$ sin reticular.

Se considera que al incorporar HIPS con LDPE se produce disminución de las propiedades mecánicas en los compuestos, principalmente en los que no están entrecruzados. El aumento en el contenido de la fibra disminuye la ductilidad del material.

Finalmente, en la curva de tensión contra deformación de los compuestos formulados con fibra natural (Figura 6a, b) se aprecia como la incorporación de fibra disminuye las propiedades de tensión, hay aumento de la curva en los compuestos formulados con DCP si se comparan con iguales niveles de fibra.

\section{Conclusiones}

Al mezclar HIPS con LDPE se obtuvieron probetas libres de burbujas originadas por el aire atrapado en la matriz del LDPE durante la etapa del mezclado, sin embargo hay efecto negativo en las propiedades mecánicas, especialmente para las mezclas sin entrecruzar.

Los compuestos formulados con DCP presentan valores más altos de las propiedades de tensión. Las

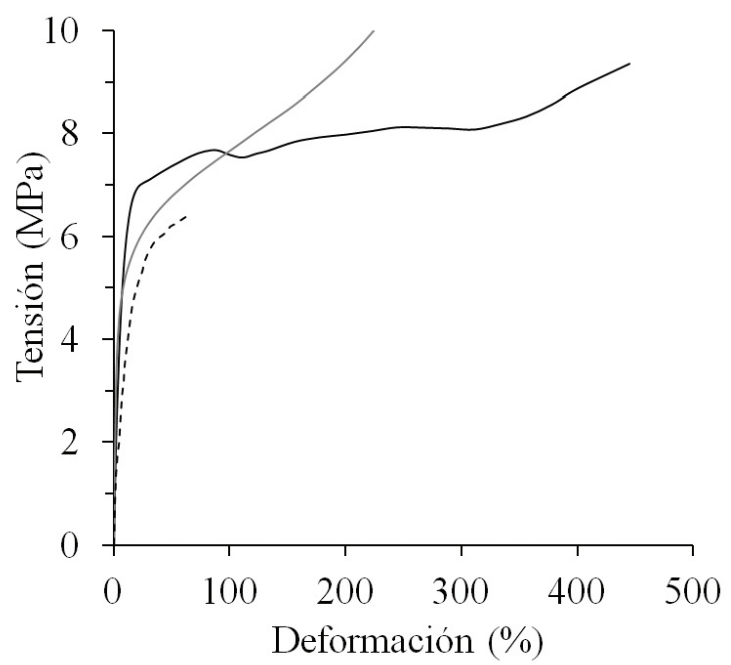

(b)

Figura 10. Ensayo de Tensión para la matriz polimérica virgen: a) sin DCP; b) con DCP. Tipo de polímeros: LDPE (—), HIPS (- - ), LDPE/HIPS (----). 
propiedades aumentan debido al entrecruzamiento de las cadenas poliméricas. Sin embargo, a medida que aumenta el contenido de fibra el efecto del DCP no es significativo debido a dos aspectos relacionados: la disminución de polímero que rodea superficialmente la partícula y la separación cada vez mayor de los sitios disponibles para producir entrecruzamientos.

La fibra natural aporta su efecto elástico al módulo de elasticidad del compuesto, la relación entre el módulo de Young y el contenido de fibra es directamente proporcional.

El porcentaje de fibra no afecta significativamente la resistencia a la fluencia.

La resistencia a la tracción, la tenacidad y la ductilidad disminuyen al aumentar la concentración de fibra. No hay efecto reforzante debido a la baja adhesión inter-facial fibra-polímero.

Investigaciones futuras serán orientadas hacía el mejoramiento de la adhesión inter-facial fibra-polímero, implementando agentes de acoplamiento y optimizando el proceso de mercerización.

\section{Referencias}

1. Nabi, D. \& Jog, J. - Adv. Polym. Technol., 18, p.351 (1999).

2. Westman, M. P.; Fifield, L. S.; Simmons, K. L.; Laddha, S. G. \& Kafentzis, T. A. - "Natural fiber composites: a review”, U. S. Departament of Energy, Washington (2010). http://dx.doi.org/10.2172/989448

3. Sullcahuamán, J. A.; Fuentes, C. A.; Mateo, M.; Pastor, A. V.; Castro, O. N. \& Zabaleta, J. S. - "Materiales compuestos de cemento, papel reciclado, quitosano y refuerzo de fibras de sisal quimicamente modificadas", in: $8^{\circ}$ Congreso Iberoamericano de Ingeniería Mecánica, Lima (2007).

4. Mihaela, D,; Nicoleta, A.; Ghiurea, M.; Ilie, C.; Radovici, C. \& Doina, M. - "Properties of Polymer Composites with Cellulose Microfibrils", en: Advances in Composites Materials-Ecodesign and Analysis, cap.5, Attaf, B (ed.), Intech Open Acces Publisher, Rijeka Croatia (2011). PMid:21567461.

5. Lee, B.; Kim, H. \& Yu, W. - Fibers Polym., 10, p.83 (2009). http://dx.doi.org/10.1007/s12221-009-0083-z

6. Rosário, F.; Pachekoski, W.; Silveira, A.; Santos, S.; Júnior, H. \& Casarin, A. S. - Polímeros, 21, p.90 (2011). http:// dx.doi.org/10.1590/S0104-14282011005000021

7. Spinacé, M.; Janeiro, L.; Bernardino, F.; Grossi, T. \& De Paoli M. - Polímeros, 21, p.168 (2011). http://dx.doi. org/10.1590/S0104-14282011005000036

8. Ngu, H. M.; Koubaa, A.; Cloutier, A.; Soulounganga, P. \& Wolcott, M. - Composites Part A: Appl. Sci. Manuf., 41, p.131 (2010).

9. Chen, H.; Chen, T. \& Hsu, C. - Holz als Roh und Werkstoff, 64, p.172 (2006). http://dx.doi.org/10.1007/ s00107-005-0072-x
10. Balam-Cocom, R. J.; Duarte, S. \& Canché, G. - Rev. Mexicana Ing. Quim., 5, p.39 (2006).

11. Cyras, V. - Rev. SAM, 2, p.18 (2005)

12. Mwaikambo, L. Y. \& Ansell, M. P. - "The effect of chemical treatment on the properties of hemp, sisal, jute and kapok fibres for composite reinforcement", in: 2nd International Wood and Natural Fibre Composites Symposium, Kassel (1999).

13. Hashim, M.; Roslan, M.; Amin, A.; Ahmad, A. \& Ariffin, S. - World. Acad. Sci. Eng. Technol., 68, p.1638 (2012).

14. Liu, L.; Yu, J.; Cheng, L. \& Qu, W. - Composites Part A: Appl. Sci. Manuf., 40, p.669 (2009). http://dx.doi. org/10.1016/j.compositesa.2009.03.002

15. Khan, J.; Khan, M. \& Islam, R. - Fibers Polym., 13, p.1300 (2012). http://dx.doi.org/10.1007/s12221-012-1300-8

16. Pires, E.; Merlini, C.; Al-Qureshi, H.; Salmória, G. \& Barra, M. - Polímeros, 22, p.339 (2012). http://dx.doi. org/10.1590/S0104-14282012005000053

17. Koning, C.; Van, D. M.; Pagnoulle, C. \& Jerome, R. - Prog. Polym. Sci., 23, p.707 (1998). http://dx.doi.org/10.1016/ S0079-6700(97)00054-3

18. Tamboli, S.; Mhaske, S. \& Kale, D. - Indian. J. Chem. Technol., 11, p.853 (2004).

19. Hieu, M. - "Compatibilization of rubber/polyethylene blends", M. Sc. Thesis, Lulea University of Technology, Sweden (2008).

20. Siong, S. - "Effects of polystyrene modified natural rubber on the properties of polypropylene -polystyrene blends", M. Sc. Thesis, University Sains Malaysia, Malaysia (2008).

21. Ghasemi, I. \& Morshedian, J. - Iranian Polym. J., 12, p.119 (2003).

22. Ahmad, E. \& Luyt, A. - Composites Part A: Appl. Sci. Manuf., 43, p.703 (2012). http://dx.doi.org/10.1016/j. compositesa.2011.12.011

23. Mokoena, M.; Djokovic, V. \& Luyt, A. - J. Materials Sci., 39, p.3403 (2004) http://dx.doi.org/10.1023/ B:JMSC.0000026943.47803.0b

24. American Society for Testing and Materials - ASTM. - "ASTM D 2765-01: Standar Test Methods for Determination of Gel Content and Swell Ratio of Crosslinked Ethylene Plastics" (2001).

25. American Society for Testing and Materials - ASTM.-“ASTM D 638-02: Standar Test Method for Tensile Properties of Plastics" (2002).

26. Betancourt, S.; Gañán, P.; Jímenez, A. \& Cruz, L. J. - Rev. Latin. Metalurgia y Materiales, 1, p.215 (2009).

27. De La Cuesta, J.; Montoya, U.; Betancourt, S. \& Álvarez, C. - Prospect, 9(2), p.75 (2011).

28. Rios, A. \& Martín, A. - An. Mecánica Fractura, 1, p.163 (2007).

Enviado: 03/07/13 Reenviado: $10 / 12 / 13$ Aceptado: 04/02/14 